\title{
Machine Learning Elman Technique for Predicting Shelf Life of Burfi
}

\author{
Sumit Goyal \\ National Dairy Research Institute, Karnal -132001, India \\ Email:thesumitgoyal@gmail.com \\ Gyanendra Kumar Goyal \\ National Dairy Research Institute, Karnal -132001, India \\ Email:gkg5878@yahoo.com
}

\begin{abstract}
Elman artificial neural network single and multilayer computerized models were developed for predicting the shelf life of burfi stored at $30^{\circ} \mathrm{C}$. The experimental data of the product relating to moisture, titratable acidity, free fatty acids, tyrosine, and peroxide value were taken as input variables, and overall acceptability score as output variable for developing the models. Bayesian regularization algorithm was applied as training algorithm for neural network. Transfer function for hidden layers was tangent sigmoid; while for output layer it was pure linear function. Elman model with a combination of $5 \rightarrow 10 \rightarrow 1$ and $5 \rightarrow 7 \rightarrow 7 \rightarrow 1$ performed exceedingly well for predicting the shelf life of burfi.
\end{abstract}

Index Terms - Elman, Artificial Neural Network, Artificial Intelligence, Burfi, Shelf Life Prediction

\section{INTRODUCTION}

The first Artificial Neural Network (ANN), called perceptron was invented in the year 1958 by psychologist Frank Rosenblatt. It was intended to model how the human brain processed visual data and learned to recognize objects. An ANN operates by creating connections between many different processing elements, each analogous to a single neuron in a biological brain. These neurons may be physically constructed or simulated by a digital computer. Each neuron takes many input signals, then, based on an internal weighting system, produces a single output signal which is sent as input to another neuron. The neurons are tightly interconnected and organized into different layers. The input layer receives the input; the output layer produces the final output [1].

Elman models are two layered backpropagation networks, with the addition of a feedback connection from the output of the hidden layer to its input. This feedback path allows Elman model to learn to recognize and generate temporal patterns, as well as spatial patterns The Elman ANN model has tansig neurons in its hidden layer, and purelin neurons in its output layer. This combination is special in that two layered networks with these transfer functions can approximate any function (with a finite number of discontinuities) with arbitrary accuracy. Elman model differs from conventional two layer networks in that the first layer has a recurrent connection. The delay in this connection stores values from the previous time step, which can be used in the current time step. Therefore, even if two Elman models, with the same weights and biases, are given identical inputs at a given time step, their outputs can be different because of different feedback states. The Elman models can be trained to respond to, and to generate, both kinds of patterns [2].

Burfi is very popular sweet confection prepared from water buffalo milk. In Indian subcontinent burfi is essentially and customarily served and consumed on all festive occasions and also during social gatherings like marriages and birthday parties. Though, several verities of burfi such as cashew nut burfi, coconut burfi, chocolate burfi, almond burfi, pistachio burfi, cardamom burfi and plain burfi are sold in the market, but the latter variety is most popular which contains milk solids and sugar. Burfi is coated with an edible thin metallic silver leaf for making the product more appealing.

Shelf life is the length of time that a product is acceptable and meets the consumer's expectations regarding food quality. Shelf life dating is one of the most difficult tasks for food industry. Market pressure has led to the implementation of shelf life by sensory analyses, which may not reflect the full quality spectra. Moreover, traditional methods for shelf life dating and small scale distribution chain tests cannot reproduce in a laboratory the real conditions of storage, distribution, and consumption on food quality. The food engineers are facing the challenges to monitor, diagnose, and control the quality and safety of food products [3].

The ANN models for predicting the shelf life of many food products have been reported. Goyal and Goyal [4] developed brain based artificial intelligent scientific computing models for shelf life detection of cakes stored at $30^{\circ} \mathrm{C}$. The potential of simulated neural networks for estimating the shelf life of soft cakes stored at $10^{\circ} \mathrm{C}$ was implemented [5]. Cascade models were suggested for predicting the shelf life of Kalakand, a desiccated sweetened dairy product [6]. For forecasting the shelf life of instant coffee drink, radial basis artificial neural engineering and multiple linear regression models were proposed [7]. Cascade forward and feedforward backpropagation artificial intelligence models for determination of sensory quality of instant coffee 
flavoured sterilized drink have been evolved [8]. Neuron based artificial intelligent scientific computer engineering models for estimating the shelf life of instant coffee sterilized drink have been reported [9]. ANNs for estimating the shelf life of milky white dessert jeweled with pistachio were implemented by Goyal and Goyal [10]. Time-delay and linear layer (design) intelligent computing expert system models predicted the shelf life of soft mouth melting milk cakes stored at $6^{\circ} \mathrm{C}$ [11]. Also, the shelf life of brown milk cakes decorated with almonds was estimated by radial basis (exact fit) and radial basis (fewer neurons) ANN models [12].

A series of partial least squares (PLS) models were employed to correlate spectral data from FTIR analysis with beef fillet spoilage during aerobic storage at different temperatures, viz., $0,5,10,15$ and $20^{\circ} \mathrm{C}$ [13].The performance of the PLS models was compared with a three - layer feedforward ANN developed using the same dataset. FTIR spectra were collected from the surface of meat samples in parallel with microbiological analyses to enumerate the total viable counts. Sensory evaluation was based on a three-point Hedonic scale classifying meat samples as fresh, semi-fresh, and spoiled. The purpose of the modelling approach employed in this work was to classify beef samples in the respective quality class as well as to predict their total viable counts directly from TIR spectra. The results obtained demonstrated that both approaches showed good performance in discriminating meat samples in one of the three predefined sensory classes. The PLS classification models showed performances ranging from 72.0 to $98.2 \%$ using the training dataset, and from 63.1 to $94.7 \%$ using independent testing dataset. The ANN classification model performed equally well in discriminating meat samples, with correct classification rates from 98.2 to $100 \%$ and 63.1 to $73.7 \%$ in the train and test sessions, respectively. PLS and ANN approaches were also applied to create models for the prediction of microbial counts. The performance of these was based on graphical plots and statistical indices (bias factor, accuracy factor and root mean square error).

A physical- empirical model to describe heat transfer of helical coil in oil and glycerol/water solution was designed [14]. It included an ANN model working with equations of continuity, momentum and energy in each flow. The discretized equations were coupled using an implicit step by step method. The natural convection heat transfer correlation based on ANN was developed and evaluated. In this ANN Prandtl number, Rayleigh number, helical diameter and coils turns number were used as input parameters; and Nusselt number as output parameter. The best ANN model was obtained with four neurons in the hidden layer with good agreement ( $\mathrm{R}>$ 0.98). Helical coil used hot water for the inlet flow; heat transfer by conduction in the internal tube wall was also considered. The simulated outlet temperature was carried out and compared with the experimental database in steady-state. The numerical results for the simulations of the heat flux, for these 91 tests in steady-state, had a $\mathrm{R} \geq$ 0.98 with regard to experimental results. This ANN correlation was proposed to predict natural convection heat transfer coefficient from helical coil for both fluids: oil and glycerol/water solution, thus saving time and improving general system performance.

ANN model was developed for the prediction of viscosity of fruit juice as a function of concentration and temperature. The fruit juices considered in the reported study were orange, peach, pear, malus floribunda and black current [15]. The viscosity data of juices (1.53$3300 \mathrm{mPas}$ ) were obtained from the literature for a wide range of concentration $\left(5-70^{\circ} \mathrm{Brix}\right)$ and temperature $\left(30.7-71.7^{\circ} \mathrm{C}\right)$. Several configurations were evaluated while developing the optimal ANN model. The optimal ANN model consisted of two hidden layers with two neurons in each hidden layer. This model was able to predict viscosity with a mean absolute error of $3.78 \mathrm{mPas}$. The performance of the ANN was checked using experimental data. Predicted viscosity using the ANN proved to be a simple, convenient and accurate method. The model can be incorporated in the heat transfer calculations during fruit processing where concentration and temperature dependent viscosity values are required. This may also be useful in mass transfer calculations during filtration of the juice using membranes for clarification.

ANN modeling and several mathematical models were applied to predict the moisture ratio in an apple drying process. Four drying mathematical models were fitted to the data obtained from eight drying runs and the most accurate model was selected. Two sets of ANN modeling were also performed [16]. . In the first set, the data obtained from each pilot were modeled individually to compare the ANN predictions with the best mathematical model. In the second set of ANN modeling, the simultaneous effect of all the four input parameters including air velocity, air temperature, the thickness of apple slices and drying time was investigated. The results showed that the ANN predictions were more accurate in comparison with the best fitted mathematical model. In addition, none of the mathematical models were able to predict the effect of the four input parameters simultaneously, while the presented ANN model predicted this effect with a good precision.

Fathi et. al. [17] studied ANN to predict the mass transfer kinetics and color changes of osmotically dehydrated kiwifruit slices. Kiwifruits were dehydrated implementing four different sucrose concentrations, at three processing temperatures and during four osmotic time periods. A multilayer ANN was developed by using the operation conditions as inputs to estimate water loss, solid gain, and color changes. It was found that ANN with 16 neurons in hidden layer gives the best fitting with the experimental data, which made it possible to predict solid gain, water loss, and color changes with acceptable mean-squared errors $(1.005,2.312$, and 2.137, respectively). The results showed that ANN could potentially be used to estimate mass transfer kinetics and color changes of dehydrated kiwifruit. 
Freeze-drying of foamed and non foamed apple juice was studied in order to assess if there is a reduction in process time due to foaming. Foams were prepared by whipping apple juice with methylcellulose or egg albumin at different concentrations [18]. Foamed and non foamed juice samples having different thickness and different initial weight were frozen at $-40^{\circ} \mathrm{C}$ and then freeze dried at $20^{\circ} \mathrm{C}$ during $48 \mathrm{~h}$ under vacuum. Sample weight loss and temperature were followed at different process times. A mathematical model based on ANNs was developed to represent foam kinetics and temperature curves during freeze-drying. This study revealed that freeze-drying of foamed materials is limited by heat transfer, while for non foamed ones, by mass transfer. It was shown that the insulation property characteristic of foams was more significant in slowing down the freeze-drying process than the increased surface area available for mass transfer due to foaming. The outcome of the study was that ANN can be used for excellent predictions of moisture content and temperature during the freeze-drying process.

The objective of the work reported in this communication is to propose Elman single and multilayer ANN models for estimating the shelf life of burfi stored at $30^{\circ} \mathrm{C}$. Findings of this study will be very useful to food industry in general and dairy industry in particular, besides wholesalers, retailers, consumers, regulatory authorities, food researchers, and academicians.

\section{METHOD AND MATERIAL}

For developing Elman ANN models, the experimental data of the product relating to moisture, titratable acidity (TA), free fatty acids (FFA), tyrosine, and peroxide value (PV) were taken as input variables; while overall acceptability score (OAS) as output variable (Fig.1).

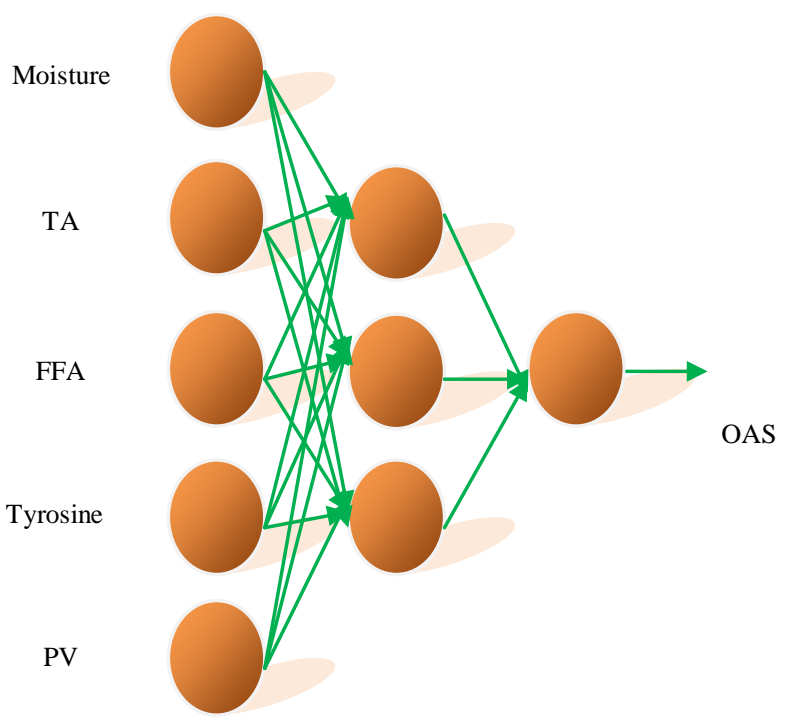

For developing the models 48 observations were included in the study. The data was randomly divided into two disjoint subsets, viz., training set having 40 observations and validation set 8 observations. Mean square error (MSE) (1), Root mean square error (RMSE) (2), Coefficient of determination: $R^{2}$ (3) and Nash Sutcliffo coefficient: $E^{2}$ (4) were applied in order to compare the prediction ability of the developed models.

$M S E=\left[\sum_{1}^{N}\left(\frac{Q_{\exp }-Q_{c a l}}{n}\right)^{2}\right]$

$R M S E=\sqrt{\frac{1}{n}\left[\sum_{1}^{N}\left(\frac{Q_{\text {exp }}-Q_{\text {cal }}}{Q_{\exp }}\right)^{2}\right]}$

$R^{2}=1-\left[\sum_{1}^{N}\left(\frac{Q_{\text {exp }}-Q_{c a l}}{Q_{\text {exp }}^{2}}\right)^{2}\right]$

$E^{2}=1-\left[\sum_{1}^{N}\left(\frac{Q_{\exp }-Q_{c a l}}{Q_{\exp }-\overline{Q_{\exp }}}\right)^{2}\right]$

Where,

$Q_{\text {exp }}=$ Observed value; $Q_{\text {cal }}=$ Predicted value;

$\bar{Q}_{\text {exp }}=$ Mean predicted value; $n=$ Number of observations in dataset.

\section{RESULTS AND DISCUSSION}

The performance matrices of ANN models for predicting the OAS are presented in Table I and II.

Figure1. Input and output variables for ANN 
TABLE I. RESULTS OF ELMAN SINGLE LAYER MODEL

\begin{tabular}{|c|c|c|c|c|}
\hline Neurons & MSE & RMSE & $R^{2}$ & $\mathrm{E}^{2}$ \\
\hline 3 & $4.29778 \mathrm{E}-06$ & 0.002073109 & 0.997926891 & 0.999995702 \\
\hline 4 & $6.92317 \mathrm{E}-05$ & 0.008320558 & 0.991679442 & 0.999930768 \\
\hline 5 & $3.55478 \mathrm{E}-05$ & 0.005962196 & 0.994037804 & 0.999964452 \\
\hline 6 & $5.48866 \mathrm{E}-05$ & 0.007408551 & 0.992591449 & 0.999945113 \\
\hline 7 & $7.02382 \mathrm{E}-05$ & 0.008380822 & 0.991619178 & 0.999929762 \\
\hline 8 & $6.54732 \mathrm{E}-05$ & 0.008091551 & 0.991908449 & 0.999934527 \\
\hline 9 & $4.55128 \mathrm{E}-06$ & 0.002133373 & 0.997866627 & 0.999995449 \\
\hline 10 & $8.53887 \mathrm{E}-09$ & $9.2406 \mathrm{E}-05$ & 0.999907594 & 0.999999991 \\
\hline 11 & $4.13281 \mathrm{E}-06$ & 0.002032932 & 0.997967068 & 0.999995867 \\
\hline 12 & $5.03415 \mathrm{E}-05$ & 0.007095174 & 0.992904826 & 0.999949659 \\
\hline 13 & $2.08673 \mathrm{E}-05$ & 0.004568071 & 0.995431929 & 0.999979133 \\
\hline 14 & $3.37114 \mathrm{E}-06$ & 0.001836067 & 0.998163933 & 0.999996629 \\
\hline 15 & $6.52134 \mathrm{E}-05$ & 0.008075481 & 0.991924519 & 0.999934787 \\
\hline 16 & $2.83382 \mathrm{E}-06$ & 0.001683396 & 0.998316604 & 0.999997166 \\
\hline 17 & $2.51848 \mathrm{E}-06$ & 0.001586973 & 0.998413027 & 0.999997482 \\
\hline 18 & $2.36328 \mathrm{E}-07$ & 0.000486136 & 0.999513864 & 0.999999764 \\
\hline 19 & $4.1013 \mathrm{E}-05$ & 0.006404138 & 0.993595862 & 0.999958987 \\
\hline 20 & $2.71339 \mathrm{E}-06$ & 0.001647237 & 0.998352763 & 0.999997287 \\
\hline
\end{tabular}

TABLE II. RESULTS OF ELMAN MULTILAYER MODEL

\begin{tabular}{|c|c|c|c|c|}
\hline Neurons & MSE & RMSE & $\mathrm{R}^{2}$ & $\mathrm{E}^{2}$ \\
\hline $3: 3$ & $2.33084 \mathrm{E}-06$ & 0.001526708 & 0.998473292 & 0.999997669 \\
\hline $4: 4$ & $4.65472 \mathrm{E}-06$ & 0.002157479 & 0.997842521 & 0.999995345 \\
\hline $5: 5$ & $3.06064 \mathrm{E}-05$ & 0.005532307 & 0.994467693 & 0.999969394 \\
\hline $6: 6$ & $5.72936 \mathrm{E}-05$ & 0.007569257 & 0.992430743 & 0.999942706 \\
\hline $7: 7$ & $1.45274 \mathrm{E}-10$ & $1.2053 \mathrm{E}-05$ & 0.999987947 & 1 \\
\hline $8: 8$ & $3.13668 \mathrm{E}-05$ & 0.005600607 & 0.994399393 & 0.999968633 \\
\hline $9: 9$ & $3.16776 \mathrm{E}-06$ & 0.00177982 & 0.99822018 & 0.9999966832 \\
\hline $10: 10$ & $3.99903 \mathrm{E}-05$ & 0.006323785 & 0.993676215 & 0.99996001 \\
\hline $11: 11$ & $1.32956 \mathrm{E}-06$ & 0.001153066 & 0.998846934 & 0.99999867 \\
\hline $12: 12$ & $1.00739 \mathrm{E}-07$ & 0.000317395 & 0.999682605 & 0.999999899 \\
\hline $13: 13$ & $3.09666 \mathrm{E}-06$ & 0.001759732 & 0.998240268 & 0.9999966903 \\
\hline $14: 14$ & $9.65734 \mathrm{E}-05$ & 0.009827177 & 0.990172823 & 0.999903427 \\
\hline $15: 15$ & $2.89837 \mathrm{E}-07$ & 0.000538365 & 0.999461635 & 0.999999971 \\
\hline $16: 16$ & $3.16776 \mathrm{E}-06$ & 0.00177982 & 0.99822018 & 0.9999996832 \\
\hline $17: 17$ & 0.000130558 & 0.011426203 & 0.988573797 & 0.999869442 \\
\hline $18: 18$ & 0.000107945 & 0.010389649 & 0.989610351 & 0.999892055 \\
\hline $19: 19$ & $3.23967 \mathrm{E}-06$ & 0.001799908 & 0.998200092 & 0.99999676 \\
\hline $20: 20$ & $2.43015 \mathrm{E}-05$ & 0.004929659 & 0.995070341 & 0.999975698 \\
\hline
\end{tabular}




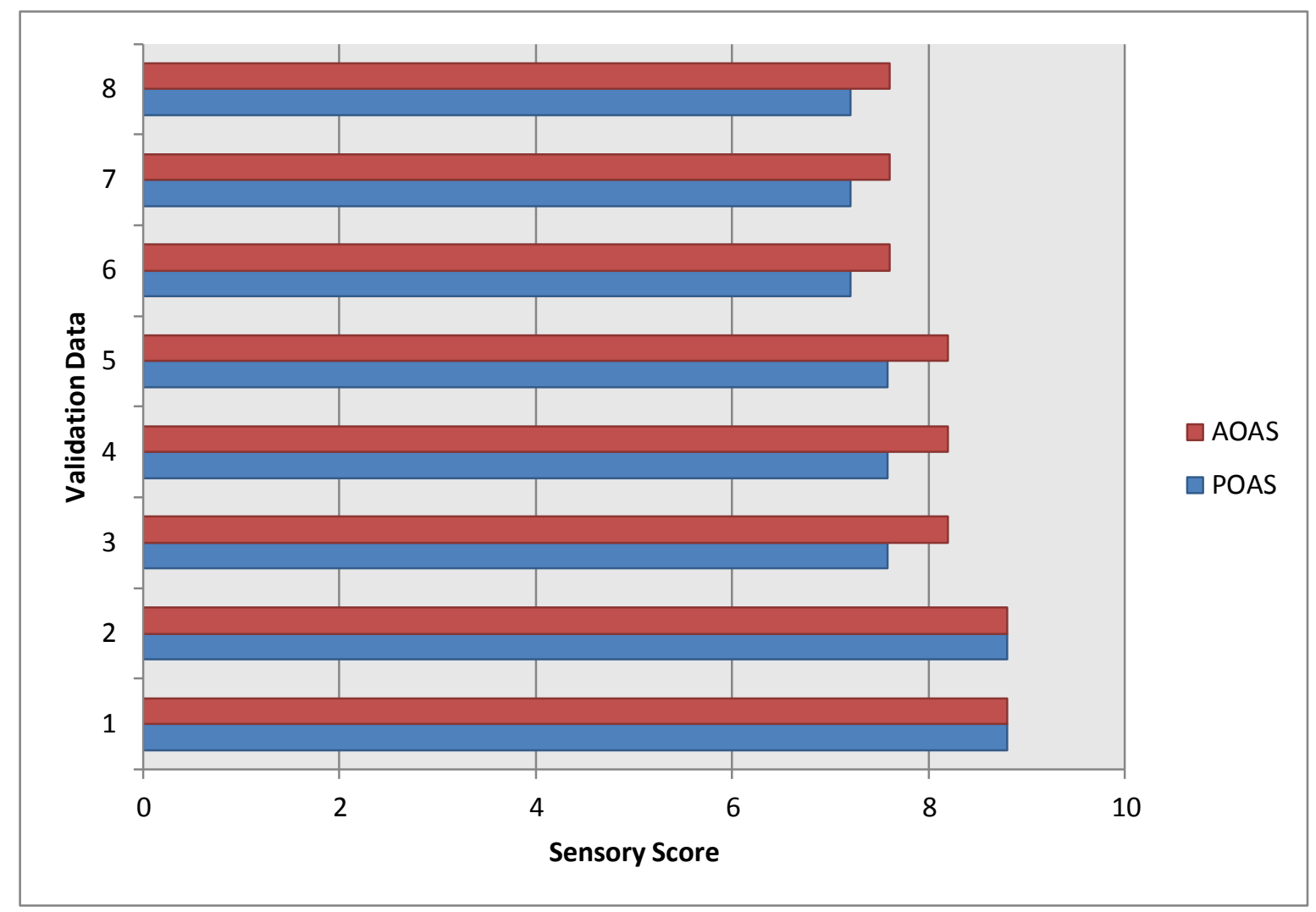

Figure 2. Comparsion of AOAS and POAS for Elman single layer model

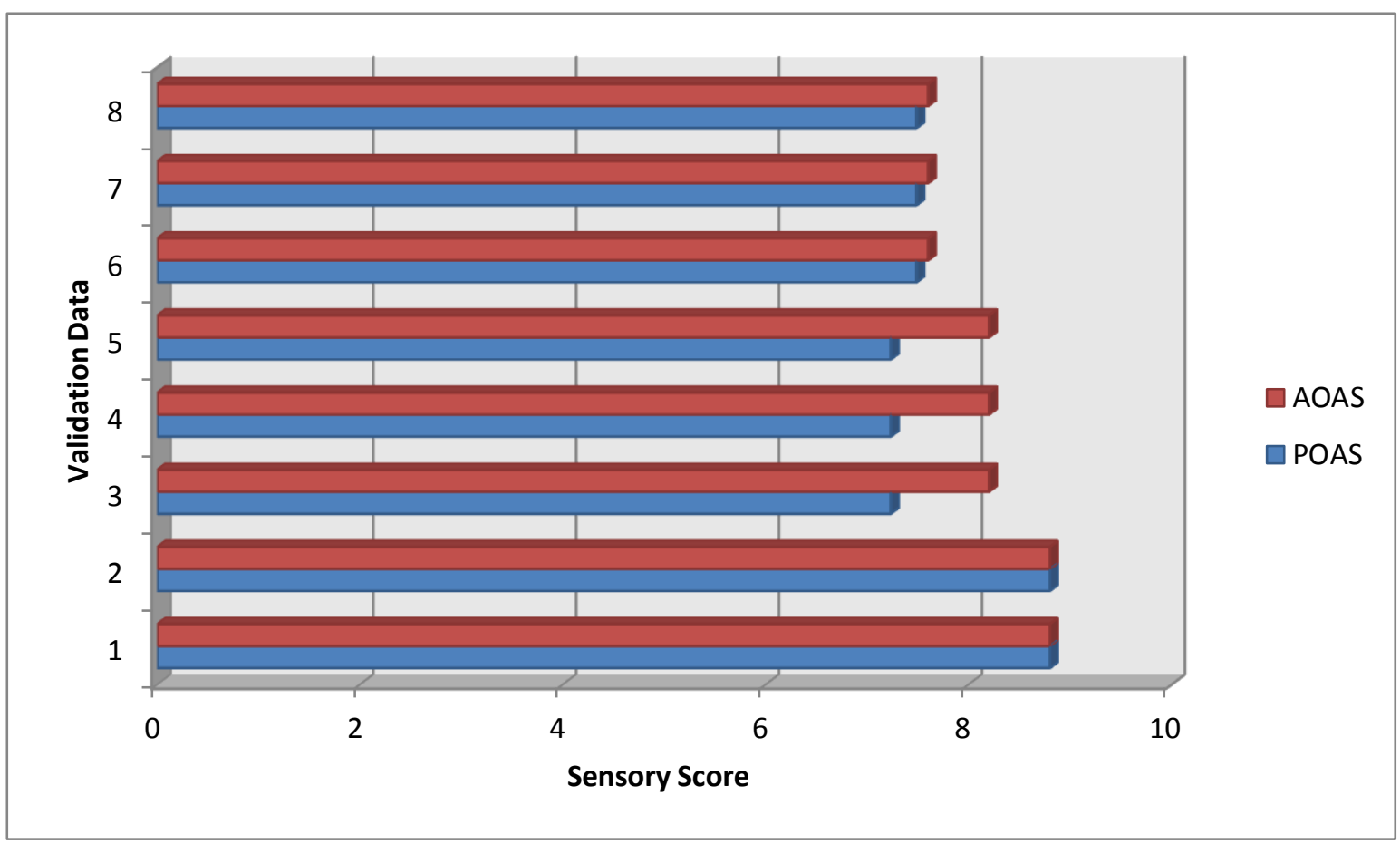

Figure 3. Comparsion of AOAS and POAS for Elman multilayer model 
The comparison of Actual overall acceptability score (AOAS) and Predicted overall acceptability score (POAS) for Elman models are illustrated in Fig.2 and Fig.3. Elman models with single and multiple hidden layers were developed and compared with each other. Bayesian regularization algorithm was used for training the network, and the transfer function for hidden layers was tangent sigmoid, while for the output layer it was pure linear function. The ANN was trained up to 100 epochs, and neurons in each hidden layers varied from 3 to 20 . The best results were achieved for single layer Elman model with the combination of $5 \rightarrow 10 \rightarrow 1$, with high coefficient of determination (0.999907594) and Nash Sutcliffo coefficient (0.999999991) (Table I). The best results for the multilayer Elman model were for the combination of $5 \rightarrow 7 \rightarrow 7 \rightarrow 1$, with high $\mathrm{R}^{2} 0.999987947$ and $\mathrm{E}^{2} 1$ (Table II), reflecting that the developed Elman ANN computerized models got very well simulated, and can be used to estimate the shelf life of burfi stored at $30^{\circ} \mathrm{C}$.

\section{CONCLUSION}

Elman single and multilayer models were developed for predicting the shelf life of burfi stored at $30^{\circ} \mathrm{C}$. The input variables were the experimental data of the product relating to moisture, titratable acidity, free fatty acids, tyrosine, and peroxide value; while overall acceptability score was output variable. The investigation revealed very good correlation between the actual overall acceptability score and the predicted overall acceptability score, with a high determination coefficient and NashSutcliffo coefficient, establishing that the developed models were able to analyze non-linear multivariate data with excellent performance. From the study it is concluded that the developed procedure which is simple, reliable, quick and low-cost is better alternative to expensive, time-consuming and cumbersome laboratory testing method for estimating the shelf life of burfi.

\section{REFERENCES}

[1] Computerworld Website, 2011: http://www.computerworld.com/s/article/57545/Arti ficial_Neural_Networks (accessed on 3.1.2011).

[2] H.Demuth, M.Beale, M. Hagan - Neural network toolbox user's guide, The MathWorks Inc., Natrick, USA, 2009.

[3] R.C.Martins, V.V. Lopes, A.A. Vicente, and J.A. Teixeira," Computational shelf-life dating: complex systems approaches to food quality and safety," Food and Bioprocess Technology, vol.1, no.3, pp. 207-222, 2008.

[4] Sumit Goyal and G.K. Goyal,'Brain based artificial neural network scientific computing models for shelf life prediction of cakes," Canadian Journal on Artificial Intelligence, Machine Learning and Pattern Recognition, vol. 2, no.6, pp.73-77, 2011.

[5] Sumit Goyal and G.K. Goyal,'Simulated neural network intelligent computing models for predicting shelf life of soft cakes," Global Journal of Computer Science and Technology, vol.11, no.14, version 1.0, pp. 29-33, 2011.

[6] Sumit Goyal and G.K. Goyal,"Advanced computing research on cascade single and double hidden layers for detecting shelf life of kalakand: An artificial neural network approach," International Journal of Computer Science \& Emerging Technologies, vol.2, no.5, pp.292-295, 2011.

[7] Sumit Goyal and G.K. Goyal,"Application of artificial neural engineering and regression models for forecasting shelf life of instant coffee drink," International Journal of Computer Science Issues, vol.8(4), no.1, pp. 320-324, 2011.

[8] Sumit Goyal and G.K. Goyal,"Cascade and feedforward backpropagation artificial neural networks models for prediction of sensory quality of instant coffee flavoured sterilized drink," Canadian Journal on Artificial Intelligence, Machine Learning and Pattern Recognition, vol.2, no.6, pp. 78-82, 2011.

[9] Sumit Goyal and G.K. Goyal,'Development of neuron based artificial intelligent scientific computer engineering models for estimating shelf life of instant coffee sterilized drink," International Journal of Computational Intelligence and Information Security, vol.2, no.7, pp.4-12,2011.

[10] Sumit Goyal and G.K. Goyal,"A new scientific approach of intelligent artificial neural network engineering for predicting shelf life of milky white dessert jeweled with pistachio," International Journal of Scientific and Engineering Research, vol. 2, no.9, pp. 1-4, 2011.

[11] Sumit Goyal and G.K. Goyal,'Development of intelligent computing expert system models for shelf life prediction of soft mouth melting milk cakes," International Journal of Computer Applications, vol.25, no.9, pp.41-44,2011.

[12] Sumit Goyal and G.K. Goyal,"Radial basis artificial neural network computer engineering approach for predicting shelf life of brown milk cakes decorated with almonds," International Journal of Latest Trends in Computing, vol. 2, no.3, pp.434-438, 2011.

[13]Z.P. Efstathios, R.M. Fady, A.A. Argyria, M.B. Conrad and E. N. George-John,"A comparison of artificial neural networks and partial least squares modelling for the rapid detection of the microbial spoilage of beef fillets based on Fourier transform infrared spectral fingerprints," Food Microbiology, vol.28, no.4, pp.782-790, 2011.

[14]D. Colorado, M.E. Ali, O. García-Valladares, O. and J.A. Hernández,'Heat transfer using a correlation by neural network for natural convection from vertical helical coil in oil and glycerol/water solution," Energy, vol.36, pp. 854-863, 2011.

[15]P. Rai, G.C. Majumdar, S. DasGupta and S. De," Prediction of the viscosity of clarified fruit juice using artificial neural network: a combined effect of concentration and temperature," Journal of Food Engineering, vol. 68, pp. 527-533, 2005. 
[16] A. Khoshhal, A.A. Dakhel, A. Etemadi and S. Zereshki," Artificial neural network modeling of apple drying process," Journal of Food Process Engineering, vol.33, pp. 298-313, 2010.

[17] M. Fathi, M. Mohebbi and S.M.A. Razavi," Application of Image Analysis and Artificial Neural Network to Predict Mass Transfer Kinetics and Color Changes of Osmotically Dehydrated Kiwifruit," Food Bioprocess Technology, vol. 4, no.8, pp.1357-1366, 2009.

[18] N. Raharitsifa and C. Ratti," Foam-mat freezedrying of apple juice part 1: Experimental data and ANN simulations," Journal of Food Process Engineering, vol. 33, pp. 268-283, 2010.

\section{AUTHOR'S BIODATA}

Sumit Goyal is M. Phil in Computer Science, Master of Computer Applications and Bachelor of Information Technology. His research interests have been in the area of soft computing, artificial neural networks and prediction of shelf life of food products. His research has appeared in Canadian Journal on Artificial Intelligence, Machine Learning and Pattern Recognition, Int. J. of Computer Applications, Int. J. of Computational Intelligence and Information Security, Int. J. of Latest Trends in Computing, Int. J. of Scientific and
Engineering Research, Int. J. of Computer Science Issues, Int. J. of Computer Science \& Emerging Technologies, Global Journal of Computer Science and Technology, Int. J. of Artificial Intelligence and Knowledge Discovery, amongst others. He is member of IDA.

Gyanendra Kumar Goyal obtained his Ph.D. degree in 1979 from Panjab University, Chandigarh, India. He was recipient of United Nations fellowship award and World Bank's fellowship award. He was also awarded Belgian Government's fellowship award. In 1985-86, he did specialized research work on Dairy and Food Packaging at Michigan State University, East Lansing, U.S.A.; and in the year 1999 he received advanced training in Education Technology at Cornell University, New York, U.S.A. His research interests include dairy \& food packaging and shelf life determination of food products. He has published more than 150 research papers in national and international journals, and presented his work in national and international conferences. His research work has been published in Int. J. of Food Sci. Technol. and Nutrition, Nutrition and Food Science, Milchwissenschaft, American Journal of Food Technology, British Food Journal, Canadian Journal on Artificial Intelligence, Int. J. of Scientific and Engineering Research, Int. J. of Computer Science Issues, Int. J. of Computer Science \& Emerging Technologies, amongst others. He is life member of AFST (I) and IDA. 